Horizons philosophiques

\title{
Comme en un miroir : quelques remarques historiques sur l'arbitraire du signe
}

\section{François Latraverse}

Volume 1, numéro 1, automne 1990

Sémiotiques 1 : mises au point, mises en question

URI : https://id.erudit.org/iderudit/800858ar

DOI : https://doi.org/10.7202/800858ar

Aller au sommaire du numéro

Éditeur(s)

Collège Édouard-Montpetit

ISSN

1181-9227 (imprimé)

1920-2954 (numérique)

Découvrir la revue

Citer cet article

Latraverse, F. (1990). Comme en un miroir : quelques remarques historiques sur l'arbitraire du signe. Horizons philosophiques, 1(1), 1-22.

https://doi.org/10.7202/800858ar d'utilisation que vous pouvez consulter en ligne.

https://apropos.erudit.org/fr/usagers/politique-dutilisation/ 


\section{Comme en un miroir: quelques remarques historiques sur l'arbitraire du signe}

C'est avec Ferdinand de Saussure que le principe de l'arbitraire du signe a été établi de manière définitive pour notre modernité sémiotique. Le maître de Genève l'a dit: le signe - linguistique, comme le commandait son propos - n'est motivé ni par un rapport de ressemblance avec ce qu'il signifie ou ce qu'il désigne, avec son concept ou son objet, ni par un rapport où on pourrait retracer une action causale directe. Ainsi, ce que nous appelons "cheval» pourrait être "Pferd» ou "enclume», «hlupák» ou "chicorée» puisqu'il ne suffit pas de regarder la bête au fond des yeux pour découvrir le nom qui lui convient. Entre le signifiant /cheval/ et le signifié "cheval», le rapport est au contraire non motivé, arbitraire, conventionnel, délibéré, artificiel, institué, etc. Le principe de l'arbitraire est cependant affirmé moins pour dégager les langues naturelles d'une causalité venant de la nature que pour diriger l'attention vers leur organisation systématique, une approche structurale ("La langue ne connaît que des différences", "Elle est faite d'oppositions internes», “Elle est un système», etc.) n'étant en effet possible que lorsqu'on a arraché la langue à l'empire des choses pour la livrer à celui des systèmes autonomes. La linguistique qu'inaugure la formulation de ce principe a ainsi pour tâche de caractériser les divers sous-systèmes constitutifs des langues en ne faisant appel précisément qu'à ce qui en fait des systèmes ${ }^{1}$. 
Malgré le caractère assez fracassant qu'elle a eu², cette affirmation de l'arbitraire du signe est pratiquement aussi ancienne que le questionnement sémiotique car on ne trouve guère, tout au long de l'histoire de la discipline, de penseur qui ne l'ait agitée d'une manière ou d'une autre. Le but de cette courte note est de récapituler quelquesunes des coordonnées de cette idée, en insistant sur certaines de ses complications philosophiques. Je chercherai en particulier à identifier les effets qu'a une conception mentaliste de la signification pour la notion de signes arbitraires.

Une des questions récurrentes au cours de cette histoire est celle du fondement de la relation de signification qui peut être traitée en termes d'origine ou en termes de fonctionnement. Dans le premier cas, on s'intéressera à la source de l'instauration du signe et on pourra procéder à une classification des signes selon qu'ils sont ou non $œ u v r e$ humaine. On l'observera ici sur un segment temporel relativement court - celui qui correspond aux positions de Port-Royal, de Condillac et surtout de Locke - mais cette approche occupe en fait la majeure partie du territoire historique. Dans le second cas, on s'attachera plutôt à caractériser différents types majeurs de signes en tenant compte de la nature du lien qui les unit à ce qu'ils désignent ou signifient. Par exemple, la classification peircienne la

1. C'est du reste pourquoi deux des possibilités lexicales que j'ai portées plus haut sont déviantes en français ou, à tout le moins, perçues comme déviantes par les locuteurs du français, pour lesquels il y a une distance considérable entre un profil lexical allemand ou tchèque (qu'on ne croie pas pour autant que "hlusák» est le nom tchèque du cheval) et l'allure qu'ont habituellement les lexèmes français. Les deux autres possibilités sont d'un autre type: l'une s'apparente partiellement à "cheval» pour une part mineure de sa phonologie, l'autre selon un rapport sémantique oblique à l'intérieur d'un univers associé aux équidés.

2. Et, sans doute, moins chez Saussure lui-même que chez ses épigones tardifs de l'épisode structuraliste qui a frappé l'Occident dans les années soixante. 
plus générale distingue les signes qui ressemblent à leurs objets (les icônes), les signes qui sont directement ou causalement affectés par eux (les index) et ceux qui sont liés à leurs objets par l'effet d'une loi (les symboles). Il est clair qu'à ce niveau de présentation, les deux approches autorisent certains recoupements, car on s'attend, par exemple, à ce que les signes dont la production est indépendante de l'activité humaine soient plutôt indexicaux, c'est-à-dire que leur occurrence soit immédiatement soumise aux contraintes des contextes où elles apparaissent ${ }^{3}$, et on s'entend en général pour reconnaître que les langues naturelles ne sont pas iconiques ${ }^{4}$.

À travers toute l'histoire de la sémiotique, la délimitation tracée entre, d'une part, les signes qui doivent d'être appelés "naturels" à leur ressemblance avec ce qu'il signifient, à la reconnaissance d'un lien causal ou simplement à l'absence d'une intervention humaine explicite, et, d'autre part, les signes établis délibérément, institués par une volonté sensible à l'économie et à la simplicité, occupe une place centrale et joue un rôle moteur. On s'aperçoit cependant vite qu'on a plus à dire des uns que des autres. II se peut qu'au sujet de signes qui ne doivent leur caractère signifiant qu'à l'existence d'une relation naturelle avec

3. Au nombre des exemples les plus courants cités à ce chapitre, on trouve la manche à air le long des pistes des aéroports, la girouette, le thermomètre, le baromètre et d'autres merveilles technologiques. Ces choses ont été fabriquées par des êtres humains et comportent à ce titre une part de convention, mais ce ne sont pas elles qui sont des signes; ce sont plutôt leurs états qui sont directement déterminés par l'orientation des vents alors en action, la température ambiante, la pression atmosphérique qui prévaut, etc.

4. De Platon à Leibniz, la réflexion philosophique s'est penchée sur le cas des onomatopées pour conclure en général que la figuration du signifié dans le signifiant n'est pas concluante parce que soutenue par des phénomènes trop variables et trop restreints. Je laisse ici de côté cette part de la recherche linguistique contemporaine qui a parfois cherché des universaux phonémiques dans les comptines enfantines, les cris des cheerleaders, les expressions de la martialité et autres formes apparentées. 
ce qu'ils signifient, une sémiotique philosophique n'ait finalement que peu de commentaires à formuler, au-delà de la reconnaissance du type de cette relation, et il est compréhensible que le gros de ses efforts porte au contraire sur ceux qui sont œuvre humaine manifeste et délibérée. L'exemple stoïcien de la cicatrice comme signe d'une blessure passée, l'exemple de tout le monde de la fumée comme signe assuré du feu ou du nuage comme signe probable de la pluie, sont des cas qui, fondés sur une relation stable dans laquelle aucune stipulation humaine n'est intervenue, fournissent la base de comparaison permettant de comprendre d'autres espèces de signes comme constituant un écart ou un ajout par rapport à une situation simple. C'est la raison pour laquelle nous, qui avons découvert la vérité de notre être dans la somme des conventions qui nous contraignent et nous constituent, sommes si doués lorsqu'il s'agit d'identifier les conditions dans lesquelles la signification non naturelle fonctionne et que nous sommes peu bavards sur celles qui régissent la signification naturelle.

II y a à cela une très bonne raison: nous ne communiquons pas au moyen de signes naturels ou, plus exactement, que lorsque nous en utilisons à nos fins de communication, ils cessent d'être naturels, pour devenir intentionnels et conventionnels. Si je fais en sorte qu'une colonne de fumée s'élève au dessus d'un feu 5 , ce ne peut évidemment être pour signaler la présence de celui-ci, mais plutôt pour signaler ma propre présence ou tout autre contenu qu'on pourra associer au fait de ce signe. Dépourvue de convention et d'intention, la colonne de fumée comme signe du feu ne laisse à penser que la nécessité causale que résume l'adage qu'il n'y a pas l'une sans l'au-

5. C'est-à-dire rien d'autre que "si j'allume un feu», mais il me fallait commencer par la fumée. 
tre ${ }^{6}$. Dans sa volonté de saisir nos opérations sémiotiques concrètes, la philosophie du langage contemporaine a consacré une copieuse part de ses efforts à définir la notion de signification non naturelle (signification $n-n$ ). H. P. Grice ${ }^{7}$, en particulier, a cherché à formuler les conditions sous lesquelles on peut dire qu'un locuteur signifie $n-n$ quelque chose. Ces conditions font d'un côté appel à l'intention dans laquelle le signe est utilisé, à la reconnaissance par autrui de cette intention, etc. ${ }^{8}$ De l'autre, elles tablent sur l'existence d'une convention institutionnelle qui permet, entre autres choses, de comprendre qu'il s'agit d'un signe pourvu d'une signification. A cette signification $n-n$ on oppose une signification naturelle qui est ainsi pensée qu'elle est sans agent émetteur et, partant, sans intention et sans convention. Cette opposition a derrière elle un certain passé dont je chercherai à retracer quelques avatars.

Les messieurs de Port-Royal introduisaient une distinction générale qui séparait les signes naturels ("comme

6. Pour dire les choses autrement, dans le cas des signes naturels, le savoir nécessaire pour les interpréter coïncide dans une large mesure avec le savoir nécessaire pour les reconnaître comme signes. Savoir que la mousse est plus abondante sur la face des arbres qui est exposée au nord est ce qui permet de savoir que cette mousse - ou l'une de ses propriétés - est un signe, ou savoir que des taches sur la peau peuvent être le signe d'une quelconque affection interne, c'est les considérer comme autre chose que des choses.

7. Les principaux textes de Grice sur ce sujet sont: "Meaning", Philosophical Review, 66, 1957, p. 377-388; “Utterer's Meaning, Sentence Meaning and Word Meaning", Foundations of Language, 4, 1968, p. 1-18; “Utterer's Meaning and Intentions", The Philosophical Review, 78, 1969, p. 147-177.

8. Cet “etc." est l'abréviation d'un certain nombre de méandres intentionnels problématiques dont B. de Cornulier dresse une carte partielle dans "Signification et non-natural meaning", Les différents types de marqueurs et la détermination des fonctions des actes de langage en contexte. Université de Genève, 1981, p. 5-22. Le diagnostic de Cornulier est que les tentatives de définir la signification $n-n$ portent plus sur une espèce de vouloir-dire privé que sur la définition d'un signe public. 
une image qui paraît dans un miroir») $)^{9}$ et ces signes «qui ne sont que d'institution et d'établissement, soit qu'ils aient quelque rapport éloigné avec la chose figurée, soit qu'ils n'en aient point du tout „10. C'est dans ce dernier cas qu'on parle habituellement d'arbitraire ${ }^{11}$. La définition que l'Encyclopédie de Diderot et d'Alembert donnera du signe un siècle plus tard demeurera port-royaliste jusque dans la lettre (il s'agit d'une reprise mot pour mot de la Logique de PortRoyal) :

"SIGNE, s.m., (métaphys.) Le signe est tout ce qui est destiné à représenter une chose. Le signe enferme deux idées, l'une de la chose qui représente, l'autre de la chose représentée; \& sa nature consiste à exciter la seconde par la première."

Cette définition se poursuit par une division des signes, en trois espèces "qui sont de plus grande utilité :

I. les signes accidentels, ou les objets que quelques circonstances particulières ont lié avec quelques-unes de nos idées, en sorte qu'ils sont propres à les réveiller. 2. les signes naturels ou les cris que la nature a établis pour les sentiments de joie, de crainte, de douleur, \&c. 3. les signes d'institution, ou ceux que nous avons nous-mêmes choisis, \& qui n'ont qu'un rapport arbitraire avec nos idées. Ces derniers signes sont nécessaires

9. Chap. 1 de La Logique ou l'Art de penser d'Arnauld et Nicole.

10. Ibid.

11. Cette notion est explicitement présente chez nombre d'auteurs et son histoire est longue. Au-delà de la question instruite dans le Cratyle de Platon, on trouve chez Aristote (traductions latines) une distinction entre les mots qui ont une base conventionnelle ("secundum placitum") et les mots "ad placitum" ou "ex arbitrio", ou encore chez Aulus Gallius, une opposition entre les "verba naturalia" et les "verba arbitraria". Le terme d'arbitraire se retrouve aussi chez plusieurs auteurs du XVI $\left.\right|^{e}$ siècle (Rabelais, Du Bellay, Pierre de la Ramée), et jusque chez Hobbes, pour qui "ex arbitrario" et "arbitrariusw remplacent pleinement "ad placitum". Wolff, dans sa "Philosophia prima sive ontologia", distingue les "signa naturalia" (qui sont "notwendig", nécessaires) et les "signa artificialia" (qui sont "willkürlich», arbitraires). 
à l'homme, pour que l'exercice de son imagination soit en son pouvoir ${ }^{12}$.

Il est une thèse partagée par Port-Royal, par les Encyclopédistes aussi bien que par la plupart des penseurs des $X V I^{e}$ et $X V I I{ }^{e}$ siècles, qui est que la nature et la fonction des signes sont de représenter moins les choses que les idées.

"Jusqu'ici, nous n'avons considéré dans la parole que ce qu'elle a de matériel, et qui est commun, au moins pour le son, aux hommes et aux perroquets. II nous reste à examiner ce qu'elle a de spirituel, qui fait un des plus grands avantages de l'homme au-dessus de tous les autres animaux, et qui est une des plus grandes preuves de la raison : c'est l'usage que nous en faisons pour signifier nos pensées, et cette invention merveilleuse de composer de vingt-cinq ou trente sons cette infinie variété de mots, qui, n'ayant rien de semblable en euxmêmes à ce qui se passe dans notre esprit, n'en laissent pas de découvrir aux autres tout le secret, et de faire entendre à ceux qui n'y peuvent pénétrer, tout ce que nous concevons, et tous les divers mouvements de notre âme ${ }^{13}$."

C'est sur la base de ces prémisses que la distinction classique est faite entre idée de chose et idée de signe :

"Quand on considère un objet en lui-même \& dans son propre être, sans porter la vue de l'esprit à ce qu'il peut représenter, l'idée qu'on en a est une idée de chose, comme l'idée de la terre, du soleil. Mais quand on ne regarde un certain objet que comme en représentant un autre, l'idée qu'on en a est une idée de signe \& ce premier objet s'appelle signe. C'est ainsi qu'on regarde d'ordinaire les cartes \& les tableaux. Ainsi le signe enferme deux idées : l'une de la chose qui représente;

12. Cette division est une reprise de celle qui est formulée dans l'Essai sur l'origine des connaissances (1.11.4) de Condillac.

13. Grammaire générale et raisonnée, deuxième partie, chapitre premier. 
l'autre de la chose représentée; \& sa nature consiste à exciter la seconde par la première ${ }^{14} \%$.

On n'est pas loin de certaines idées saussuriennes, sous deux aspects. Premièrement, le couple signifiant/signifié qui, chez Saussure, unit une image acoustique et un concept est ici rendu dans des termes voisins : «l'idée de la chose excite l'idée du son \& l'idée du son celle de la chose"; ce n'est pas un son qui est l'expression d'un sens ou d'un objet, mais l'idée d'un son qui s'associe à l'idée d'une chose pour ensemble former un signe, qui est ainsi la mise en rapport de deux idées. Deuxièmement, cette mise en rapport est arbitraire, mais il s'agit d'un arbitraire relatif, comme on le voit dans ce passage de "Apologie pour les religieuses de Port-Royal» d'Arnauld :

"Les mots ne sont pas seulement des sons, mais des signes; c'est-à-dire qu'ils ne frappent pas seulement les sens, mais qu'ils servent à former, dans l'esprit de ceux qui écoutent, les idées de certaines choses, selon qu'il a plu aux hommes d'en attacher les unes à certains mots \& les autres à d'autres; c'est pourquoi la signification des mots est arbitraire avant qu'elle soit réglée par cette institution \& cet usage; mais quand elle l'est une fois, il n'est pas permis de la changer à sa fantaisie, $\&$ on est obligé de suivre cet accord de la société humaine, comme l'appelle S. Augustin : Pacta societatis humanae».

Cela ressemble à la mise en garde de Saussure contre une conception de l'arbitraire qui y verrait la possibilité d'improvisations incessantes et de changements libres. On insiste au contraire sur la part d'obligation que comporte l'adhésion à un contrat collectif si les conditions d'intelligibilité mutuelle doivent être respectées.

Le «modèle» de la sémiotique port-royaliste n'est ni simplement bipartite (le signe + la chose) comme cela

14. La Logique ou l'Art de penser, I, iv. 
arrive parfois, ni tripartite (le signe + le sens + la chose) comme cela arrive plus souvent, mais explicitement quadripartite (le signe en tant que chose + l'idée de cette chose + l'idée d'un représenté + le représenté). La représentation qui lie ces idées est dite être "excitée» dans l'esprit et une conception de la signification qui s'aligne sur elle est clairement de nature mentaliste. Notons que, dans un esprit très cartésien, l'idée est pour Port-Royal le cas paradigmatique de l'indéfinissable : "Le mot d'ldée est du nombre de ceux qui sont si clairs qu'on ne peut les expliquer par d'autres, parce qu'il n'y en a point de plus clairs \& de plus simples ${ }^{15}$ ". La classe des idées n'est donc pas hétérogène : l'idée est l'exemple par excellence de particule élémentaire. Pour comprendre l'impact qu'une sémiotique mentaliste peut avoir sur la question de l'arbitraire, il importe cependant de ne pas confondre l'idée et l'image :

“Lors donc que nous parlons des idées, nous n'appelons point de ce nom les images qui sont peintes en la fantaisie, mais tout ce qui est dans notre esprit, lorsque nous pouvons dire avec vérité que nous concevons une chose, de quelque manière que nous la concevions. D'où il s'ensuit que nous ne pouvons rien exprimer par nos paroles, lorsque nous entendons ce que nous disons, que de cela même il ne soit certain que nous avons en nous l'idée de la chose que nous signifions par nos paroles, quoique cette idée soit quelquefois plus claire et plus distincte, et quelquefois plus obscure et plus confuse. [...] Car il y aurait de la contradiction entre dire que je sais ce que je dis en prononçant un mot, et que néanmoins je ne conçois rien en le prononçant que le son même du mot ${ }^{16}$ ".

La première phrase de la première partie de la Logique formulait déjà le principe : “[...] nous ne pouvons avoir aucune connaissance de ce qui est hors de nous que par

15. Ibid., I, 1.

16. C'est pourquoi, précisent-ils, nous avons une idée de Dieu ou d'une figure à 1294 côtés alors que nous n'en avons pas d'image. 
l'entremise des idées qui sont en nous." Ce qu'on a par la suite fustigé comme la métaphysique de l'intérieur et de l'extérieur fonctionne ici à plein rendement : nous ne nous engageons pas quant à l'existence des choses puisqu'il suffit que nous en concevions l'idée en en formant les mots et aucun critère de validation externe ne peut intervenir ${ }^{17}$. Une thèse déterminante du cartésianisme est que les impressions mécaniques causées à nos organes par les objets externes ne ressemblent pas aux idées que nous nous formons de ces objets. II est intéressant de noter que cette thèse s'appuie chez Descartes sur une analogie linguistique :

“Encore que chacun se persuade communément que les idées que nous avons en notre pensée sont entièrement semblables aux objets dont elles procèdent, je ne vois point toutefois de raison qui nous assure que cela soit; mais je remarque, au contraire, plusieurs expériences qui nous en doivent faire douter. Vous savez bien que les paroles, n'ayant aucune ressemblance avec les choses qu'elles signifient, ne laissent pas de nous les faire concevoir [...] Or, si des mots, qui ne signifient rien que par l'institution des hommes, suffisent pour nous faire concevoir des choses, avec lesquelles ils n'ont aucune ressemblance, pourquoi la Nature ne pourra-t-elle pas aussi avoir établi certain signe, qui nous fasse avoir le sentiment de la Lumière, bien que ce signe n'ait rien en soi, qui soit semblable à ce sentiment? Et n'est-ce pas ainsi qu'elle a établi les ris et les larmes, pour nous faire lire la joie et la tristesse sur le visage des hommes ${ }^{18}$ ?"

Comme l'ont fait remarquer plusieurs commentateurs, on assiste là à un renversement radical des perspectives sémiotiques antérieures, pour lesquelles le signe naturel était un idéal stable qui permettait par défaut d'identifier les signes d'institution comme étant l'œuvre de la loi et de

17. L'origine est nettement cartésienne; cf. CEuvres, (A \& T.) IXa, p. 124; p. 141, 209; III, p. 392-393.

18. CEuvres, XI, p. 3-4. 
la convention, avec une composante d'arbitraire qui les rendrait fragiles par rapport à la solidité naturelle. C'est maintenant la source d'une institution omniprésente (soit institution de Dieu - ou, ce qui revient le plus souvent au même, de la Nature - soit institution humaine) qui est le concept clé, et non plus le caractère intrinsèque ou extrinsèque, intérieur ou extérieur de la liaison instaurée. D'un point de vue iconique, le signe naturel devient aussi «arbitraire" que le signe d'institution. C'est sans doute chez Gérauld de Cordemoy et chez Louis de La Forge que cette thèse trouvera son expression la plus pure, avec une structure quadripartite bien identifiée. Descartes parle des «idées que nous avons des couleurs, des sons, des odeurs ou des goûts" et il distingue "l'idée du son" et "l'idée du mot». La Forge écrit pour sa part :

"Quand nous ne savons pas encore l'usage et la prononciation des mots, l'impression qu'ils font sur nos sens ne nous fait pas concevoir autre chose que les sons ou les figures des Lettres, mais lorsque nous l'avons apprise et que nous nous y sommes accoutumés, [...] cette impression nous donne [...] la pensée qui nous représente ces paroles en elles- mêmes, et $[. .$.$] celle de la chose qu'elles signifient { }^{1{ }^{2}}$.

La "sémiotique cartésienne" a ainsi affaire à six entités distinctes, qui complexifient une structure quadripartite par l'adjonction de «mouvements»:

signe ..... mouvement corporel ${ }^{1}$..... idée ${ }^{1} \ldots$ idé $^{2}$...... mouvement corporel ${ }^{2}$..... chose $e^{20}$
(1)
(2)
(3)
(2)
(1)

19. CEuvres philosophiques, Presses Universitaires de France, 1974, p. 162.

20. Cordemoy identifie successivement :

1. la «voix»;

2. la afaçon dont l'air, en frappant le nerf de notre oreille, ébranle notre cerveaun ;

3. lidée 1 de la voix ;

4. l'idée 2 de la chose :

5. les «impressions que les choses laissent dans le cerveau» ;

6. la chose signifiée (Discours physique de la parole, Slatkine, 1973,

p. 218-223; p. 232-239). 
Les relations (1) sont exclusivement mécaniques; les relations (2) sont naturelles et universelles car elles sont établies par la volonté divine; la relation (3) procède selon les cas d'une institution divine ou d'une institution humaine : "une image qui paraît dans un miroir est un signe naturel de celui qu'elle représente" et l'idée de cette image et l'idée de l'individu représenté ont une relation voulue par Dieu, tandis que d'autres signes, comme les mots, "ne sont que d'institution et d'établissement", et tiennent à une convention passée par l'espèce humaine en général ou propre à chaque communauté.

Revenons à la définition port-royaliste de l'Encyclopédie. Cette définition est succincte et on ne dispose d'aucune précision aussi bien sur les termes qu'elle unit que sur la nature de l'excitation. Un élément important doit cependant être mentionné : la définition du signe, mystérieuse si on la considère en elle-même, ouvre une perspective nouvelle si on l'associe à la classification des signes qui la prolonge, car le signe y est présenté moins comme l'articulation de deux idées que dans leur mise en rapport par une pratique, qui seule rend signifiante la réunion des éléments. L'institution devient ainsi action et non simplement origine. C'est cette dimension qui fait l'originalité de Condillac et des Encyclopédistes. L'article "signification", pour lequel on inscrit - il importe de le noter "gramm. et juris.", donne le ton : "acte par lequel on notifie quelque chose à une autre personne". D'un point de vue plus général, le signe du XVIIIe siècle renvoie à la signification, laquelle a pour fonction de lier entre elles des choses dissemblables. Dans ce schéma, c'est le processus lui-même qui est le plus important.

Dans son Traité des tropes, Du Marsais en donne une illustration : «À mesure qu'on nous a donné du pain et qu'on nous a prononcé le mot "pain", d'un côté le pain a gravé son image dans notre cerveau par nos yeux, et en a excité l'idée; d'un autre côté, le son du mot "pain" a fait 
aussi son impression par nos oreilles, de sorte que ces deux idées accessoires, c'est-à-dire excitées en nous en même temps, ne sauraient se réveiller séparément, sans que l'une excite l'autre". Le schéma est le suivant :

OBJET $^{1}$ (le son) IDÉE'1 IDÉE² OBJET ${ }^{2}$ (la chose)

D'un point de vue génétique, la liaison est accidentelle car elle tient à l'activation de deux choses indépendantes, mais d'un point de vue interne, c'est-à-dire sémiotique, elle est nécessaire car une idée ne saurait éveiller l'autre que dans la mesure où la première s'est constituée en signe. Les éléments que l'article «signe» reprend de Condillac font du reste chez celui-ci l'objet de quelques développements dans une perspective génétique (Condillac s'intéresse à l'origine des connaissances). Le processus de signification accidentelle est une répétition du processus de la perception : il permet de lier une idée à un objet, et cette liaison est accidentelle dans la mesure où elle ne dépend ni de la structure du sujet qui l'éprouve ni d'une institution stable. La relation naturelle de signification est du même ordre, à ceci près qu'elle repose sur l'universalité et la nécessité organique de certaines liaisons : un son sera toujours lié à un sentiment, mais les signes naturels sont acquis, c'est-à-dire qu'ils ne sont pas amenés causalement par le sentiment, mais sont plutôt partagés par la socialisation : «leur commerce réciproque leur fit attacher aux cris de chaque passion les perceptions dont il était le signe naturel21". Bien que Condillac en vienne à penser que le langage d'institution trouve son origine dans le "langage d'action", c'est le troisième groupe de signe qui est le plus important. Sa dimension fondamentale est celle de l'institution. II faut toutefois voir que les signes naturels et

21. Essai sur l'origine des connaissances, Galilée, 1973, II.I.I. 
les signes d'institution sont en quelque sorte voisins, car les premiers n'ont pas plus d'analogie avec ce qu'ils représentent que les seconds, mais ils sont séparés par une différence de taille, qui est la liberté : si les êtres humains pouvaient se servir de cris pour "retracer à [leur] gré les sentiments qu'ils expriment", les signes naturels deviendraient des signes d'institution. Dans la genèse du langage, les moments assignés aux étapes du développement reprennent ces trois classes : les signes accidentels sont les premiers, suivis des signes naturels, puis des signes arbitraires. Cette chronologie est réaliste ${ }^{22}$ et recoupe celle de l'apprentissage : comme Condillac l'écrit dans sa Grammaire : "Ce qui arrive aux enfants qui apprennent les langues est arrivé aux hommes qui les ont faites".

Avec Locke, la distinction cartésienne entre, d'une part, l'idée comme état mental, comme événement psychologique, comme une certaine "façon de penser" et, d'autre part, comme une image de quelque chose, se voit fortement modifiée. Alors que Descartes définit le second sens comme étant indépendant de l'histoire individuelle du sujet et imputable à la raison, Locke retient aussi ce second sens comme étant le plus important mais choisit de retracer l'élaboration des idées selon une logique événementielle. Le psychologisme qui en résulte (par opposition au rationalisme) affecte sérieusement une théorie de la signification, qui devient l'histoire factuelle (et, dans cette mesure, accidentelle) de la constitution des idées chez les agents. Descartes admettait un arbitraire entre les idées et les mots mais le limitait à la forme phonétique et laissait la structure sémantique à l'abri, alors qu'il y a chez Locke la possibilité d'un arbitraire des idées et d'un réalignement consécutif

22. Les $X V I^{\theta}$ et $X V I I^{e}$ siècles regorgent de récits sur le développement du langage, son origine et les diverses phases de son apprentissage, mais plusieurs sont théoriques au sens où ils ne prétendent pas retracer une évolution réelle mais seulement effectuer une reconstruction rationnelle. 
de la théorie sémantique. Cette possibilité est cependant négligée dans la construction de la théorie, ses conséquences n'étant pas exploitées comme elles pourraient l'être, et la fonction du langage en est d'abord une, instrumentale, de consignation et de communication : les hommes se servent des mots, écrit-il, «soit pour enregistrer leurs propres pensées et aider ainsi leur propre mémoire, soit pour exprimer en quelque sorte leurs idées et les dis-

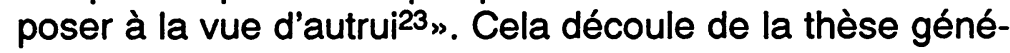
rale de Locke : les mots sont les signes des idées, les mots particuliers d'idées particulières et les mots généraux d'idées générales. Le langage a pour mission de rendre présent ce qui ne l'est pas.

"Car puisque aucune des choses que l'esprit contemple n'est, à part l'esprit lui-même, présente à l'entendement, il est nécessaire qu'une autre chose, comme signe ou représentation de la chose considérée, lui soit présente : ce sont les Idées ${ }^{24}$.»

L'esprit étant le seul objet que l'esprit puisse contempler directement, il a indirectement accès aux choses par les idées, qui sont des signes (pour cet esprit).

On doit par conséquent distinguer deux "sens" ou du moins deux "rôles" de la notion de signe : il y a d'un côté un signe instrumental pourvu d'un rôle de consignation et de communication et de l'autre un signe conceptuel (cognitif) avec une fonction de représentation du monde extérieur. Le schéma, simple, est le suivant :

23. "[...] either to record their own thoughts for the assistance of their own memory; or, as it were, to bring out their ideas, and lay them before the views of others". An Essay Concerning Human Understanding, III, 2 \$2.

24. "For since the Things, the Mind contemplates, are none of them, besides itself, present to the understanding, it is necessary that something else, as a sign or representation of the thing it considers, should be present to it : and these are Ideas." Ibid., Livre IV, chap. XXI. 
Sous ses deux espèces (celle d'idée-signe et celle de signe-signe), le signe est extérieur à la réalité, et la relation des mots aux choses est doublement médiate : si les idées sont des signes, les signes qui représentent ces signes sont des signes de signes : «parce que la scène des idées [...] ne peut être offerte à la vue immédiate d'autrui, ni dressée ailleurs que dans la mémoire" ("because the scene of ideas [...] cannot be laid open to the immediate view of another, nor laid up anywhere but in the memory"). La théorie de la signification qui en résulte est ainsi que les signes représentent directement les idées et indirectement les choses. Deux problèmes se présentent. II faut savoir premièrement à quoi rattacher la notion de signification et, deuxièmement, comment assurer l'intersubjectivité d'une sémantique idéelle.

Tout en notant les diverses manières dont des mots de catégories différentes représentent les idées ${ }^{25}$, Locke répond à la première question en affirmant que la signification est un prédicat des mots et qu'elle fonctionne en général de la même façon, à savoir en représentant des idées ${ }^{26}$. Le deuxième problème est plus sérieux et il survivra jusqu'à nous sous la question du "langage privé" traitée par Wittgenstein. Locke insiste sans cesse sur l'assimilation des significations aux idées et précise (par ex., III, 2, §3) que «les mots dans la bouche de tout homme

25. Par ex., III, $7, \$ 2$ au sujet des termes syncatégorématiques, où une distinction est faite entre cinq sens différents de la conjonction "but» et où il est dit que ules particules" "are not truly by themselves the names of any ideas".

26. Sur ce point, Berkeley apportera un correctif important : "La communication des idées exprimées par les mots n'est ni la seule ni la principale fin du langage, comme on le pense couramment". (Principes de la connaissance humaine, Aubier, p. 197). Mentionnons aussi un passage, assez curieux, où Locke, réfléchissant sur des mots comme arien» et «ignorance», en vient à conclure qu'ils signifient l'absence d'idées (III, 1, §4). 
représentent les idées qu'il $a^{27}$ ". Rien dans cette présentation n'assure que les idées d'autrui soient identiques aux miennes et l'introspection recommandée par Locke pour parvenir à la signification des mots est de peu de secours dans la résolution de cette difficulté. Si la signification que quelqu'un attache à un mot est fournie par les idées qu'il a lui-même et si «tout homme a l'insurmontable liberté de faire en sorte que les mots représentent les idées qu'il veut ${ }^{28}$ ", la conséquence s'impose qu'un langage privé (ou même «logiquement privé" comme l'a appelé Wittgenstein, c'est-à-dire un langage qui ne peut être compris que par son seul utilisateur) est possible et risque de miner toute théorie sémantique. Locke a identifié la difficulté, mais il ne la résout qu'indirectement et partiellement par un recours à l'usage commun : "l'usage commun, par un consentement tacite ${ }^{29}$, rend certains sons appropriés à certaines idées dans toutes les langues, ce qui limite la signification de ce son, de sorte qu'à défaut de l'appliquer à la même idée, on ne parle pas correctement ${ }^{30}$ ".

On joue toutefois sur deux tableaux dont la solidarité

27. "Words in every man's mouth, stand for the ideas he has". Notons au passage le singulier. Voir aussi ce fragment de III, 2, \$5, encore plus explicite : elt is perverting the use of words, and brings unavoidable obscurity and confusion into their signification, whenever we make them stand for any thing but those ideas we have in our own minds". La conséquence en est que les idées d'autrui ne sont accessibles qu'indirectement : si quelqu'un urepresents to himself other men's ideas by some of his own, if he consents to give them the same names that other men do, it is still to his own ideas, to ideas that he has, and not to ideas he has notw (III, 2, \$2).

28. a[...] every man has so inviolable liberty to make words stand for what ideas he pleases» (III, 2, 58).

29. Je souligne.

30. "[...] common use, by a tacit consent, appropriates certain sounds to certain ideas in all languages, which so far limits the signification of that sound, that unless a man applies it to the same idea, he does not speak properly" (III, 2, §8). On aura compris que le problème tient essentiellement à la fragilité des critères d'identité sur ce terrain : ce qui m'apparât être le même est-il le même? 
améliore la difficulté et la rapproche d'une certaine manière de la "solution» que Kripke a confectionnée pour le paradoxe du langage privé chez Wittgenstein ${ }^{31}$. D'une part, seules les idées propres sont susceptibles de fournir à chaque sujet la signification qu'il attache aux mots (et dont rien ne garantit qu'elle soit partagée). D'autre part, on insiste sur le fait que la pensée correcte et productive repose sur des conditions de partage linguistique supposé : les gens «supposent que leurs mots sont les marques d'idées qui se trouvent aussi dans l'esprit d'autrui32», de la même manière qu' «ils supposent souvent que les mots représentent aussi la réalité des choses ${ }^{33}$ ", à défaut de quoi, écrit Locke, «ils parleraient en vain ${ }^{34}$ ". Au-delà de la définition de la signification par les idées, il apparaît ainsi nécessaire d'assigner une signification communautaire et, dans cette mesure, objective aux mots et de contrôler les idées qu'ils

31. Cf. Saul A. Kripke, Wittgenstein On Rules and Private Language, Harvard University Press, 1982. Kripke identifie chez Wittgenstein un paradoxe sceptique relatif à l'action de suivre une règle : rien n'assure que je suive une règle si tout comportement peut être en accord avec elle et rien n'assure qu'un signe signifie un contenu déterminé si tout signe peut être mis en rapport avec n'importe quel contenu selon une règle d'interprétation quelconque. Kripke voit la solution de ce paradoxe dans les jeux de langage comme formes de l'agir commuñ : suivre une règle correctement, c'est faire comme la plupart des gens. Pour quelques commentaires sur cette question, je me permets de renvoyer à F. Latraverse «Suivre une règle : Remarques sur une lecture crypto-kripkéenne», Protée, 18:2, 1990, p. 45-54; «Remarks on the Majoritarian Account», Akten des 14. Internationalen Wittgenstein-Symposium, Vienne, Hölder-Pichler-Tempsky, 1990; et aLenguaje y comunidad», in F. Latraverse, La sombra del lenguaje, Bogotá, Presses de l'Université Nationale, sous presse. On trouvera aussi un certain nombre d'études sur cette question dans le premier numéro de la revue Lekton, consacré à Wittgenstein, Département de philosophie, Université du Québec à Montréal, 1990.

32. "[...] suppose their words to be marks of the ideas in the minds also of other men” (III, 2, \$4).

33. "[...] they often suppose the words to stand also for the reality of things" (III, 2, 55).

34. "[...] they should talk in vain» (III, 2, \$4.12) 
représentent. Le tableau que Locke laisse demeure toutefois ambigu : nous avons la liberté d'associer ce qu'il nous plaît aux mots que nous utilisons et c'est par supposition que nous pensons que ce que nous voulons dire est identique à ce qu'autrui entend, mais nous devons néanmoins faire comme autrui pour parler de manière sensée. "Les idées que nous avons dans nos esprits" et auxquelles seuls nous avons accès ne doivent d'éviter d'être des associations libres et idiosyncrasiques que si nous acceptons que l'usage les discipline causalement, usage qu'il faut parfois (se) rappeler pour éviter les confusions ${ }^{35}$.

La théorie de la signification chez Locke a comme un de ses piliers la doctrine des idées générales. Cette doctrine trouve son point de départ dans le fait que, bien que toutes choses qui existent soient particulières (individuelles), nous disposons de termes généraux dont le problème est de savoir à quoi ils correspondent. Puisque ces termes ne désignent pas des individus (auquel cas ce seraient des noms propres) et qu'ils ne désignent pas non plus une pluralité de choses, il ne reste comme possibilité que la désignation d'idées générales : «Quand des noms généraux ont quelque rapport avec des êtres particuliers, ces idées générales sont le milieu qui les unit ${ }^{36} \%$. Cette transition constitue un point assez ardu, d'autant plus que

35. A ce propos, pensons à ce que Francis Bacon écrivait déjà dans The Advancement of Learning : «Bien que nous pensions que nous gouvernons nos mots [...], il est certain que les mots [...] reviennent sur l'entendement des plus sages, prennent au piège et pervertissent le jugement. II est ainsi nécessaire dans toutes les controverses et disputes d'imiter la sagesse des mathématiciens, en établissant dès le début la définition de nos mots et de nos termes, de sorte qu'autrui puisse savoir comment nous les acceptons et les comprenons [...]. À défaut de cela, nous nous retrouverons certainement là où nous aurions dû commencer, c'est-à-dire avec des questions et des différences relatives aux mots". Des louanges analogues adressées aux mathématiciens se trouvent aussi chez Locke (IV, 3, \$30 et IV, 4, 59).

36. "[...] when general names have any connexion with particular beings, these abstract ideas are the medium that unites them" (III, $3, \&$ 13). 
Locke insiste sur les "pains and skills" qu'il faut pour former des idées générales (celle d'un triangle par exemple ${ }^{37}$ ). II en vient à la conclusion qu'une idée générale "est quelque chose d'imparfait, qui ne peut exister ${ }^{38}$ ".

La "solution" viendra de la théorie des définitions, qui permettra de voir comment une signification peut être donnée aux termes généraux. Une définition «n'est rien d'autre que la monstration ${ }^{39}$ de la signification d'un mot au moyen de plusieurs autres termes non synonymes 40 ". II s'agit de montrer l'idée dont le signe est la marque et cette monstration s'effectue par des mots qui ont pour fonction d' «annexer» l'idée à l'esprit d'autrui. Notons au passage que la distinction entre définition de signes et définition de choses, si cruciale pour Port-Royal, est ici absente : Locke exclut la définition des choses car l'essence des sortes ou des espèces des choses renvoie aux idées abstraites : "Les espèces des choses pour nous ne sont rien d'autre que le fait qu'elles sont rangées sous des noms distincts, selon les idées complexes qui sont en nous et non selon les essences réelles, précises et distinctes qui sont en elles ${ }^{41}$ ». Les idées que nous avons continuent de fournir le fin mot.

La thèse épistémologique (ou gnoséologique) générale de Locke est que toutes les idées complexes sont

\section{Cf. IV, 7, §9.}

38. "[...] is something imperfect, that cannot exist» (IV, 7, \$9). II y a ici un problème de cohérence ou d'interprétation: puisque Locke insiste par ailleurs sur le rôle fondamental des idées générales, il s'agit sans doute de l'inexistence d'objets correspondant aux idées générales. Cela tient au psychologisme (par opposition, ici encore, au rationalisme mais aussi au conceptualisme) de Locke.

39. Je souligne.

40. «[...] is nothing else but the showing the meaning of one word by several other not synonymous terms» (III, 4, \$6).

41. "[...] the species of things to us are nothing but the ranking them under distinct names, according to the complex ideas in us, and not according to precise, distinct, real essences in them” (III, 6, \$8). 
faites d'idées simples et que celles-ci nous viennent de l'expérience (que ce soit par la sensation ou la réflexion). La capacité des idées à assurer la signification a été critiquée par nombre de commentateurs de Locke; certains ont même soutenu qu'il n'avait pas de théorie de la signification (au sens de «meaning») et qu'il n'avait qu'une théorie des idées et du discours mental42. Le fait est qu'il n'utilise guère le terme «meaning" et qu'il lui préfère "sign" et "signify". Des discussions ont même été poursuivies sur la question de savoir si les mots "mean" des idées ${ }^{43}$, de manière à lever les accusations afférentes au langage privé qui ont pesé contre lui. On a sans doute intérêt ici à tirer profit de la duplicité des idées : en tant que telles, elles n'ont trait qu'à la sphère intérieure de l'individu et elles sont à ce titre privées, mais en tant que signes, elles lient l'individu à autre chose que les idées, c'est-à-dire à cette autre chose indistincte dont autrui peut aussi se former une idée, dont il devra par hypothèse supposer qu'elle est conforme à celle de ses semblables.

Dans cet espace, signes et idées se répondent sans cesse comme en un miroir et les questions importantes consistent à savoir à quoi accorder l'importance première et comment relier les idées et les signes au monde extérieur, de manière à assurer leur fiabilité. La réponse de Locke à la première question est de reconnaître le primat de l'idée et de reléguer le langage au rôle de transcription; il rejoint en cela Hobbes qui définissait comme suit la fonction principale du signe :

42. Cela est manifestement le cas chez Hobbes, qui écrit dans son Leviathan : «L'usage général du langage est de transposer notre discours mental en discours verbal, ou le flux de nos pensées en un flux de motsw (athe general use of speech, is to transfer our mental discourse, into verbal; or the train of our thoughts into a train of words" (Leviathan, 1, 4).

43. Je m'exprime ainsi parce que je n'ai pas le choix, le verbe français asignifier» correspondant aussi bien à ato signify» qu'à ato mean». 
"Les mots ainsi liés qu'ils deviennent des signes de nos pensées sont appelés adiscours» et toute part en est un nom. Mais voir (comme on dit) les marques comme les signes est nécessaire pour le développement de la philosophie (marques qui nous permettent de nous rappeler nos propres pensées, et signes qui nous permettent de les faire connaître à autrui), les noms jouent ces deux rôles; mais ils sont des marques avant que d'être des signes [...] De sorte que la nature du signe consiste principalement en cela qu'il est une aide de la mémoire; mais il sert aussi accidentellement à signifier et à rendre connu à autrui ce que nous nous rappelons nousmêmes ${ }^{44}$.

Sa réponse à la seconde question est de s'en remettre à l'usage courant dont il est manifeste qu'il n'est uni à l'usage singulier que par l'hypothèse d'une conformité. En tant que consignation du contenu de notre mémoire propre et reflet de nos idées individuelles, le langage est aléatoire. Son arbitraire ne vient plus seulement de ce qu'il a été instauré d'une manière plutôt que d'une autre, il tient aussi à ce que les idées qu'il représente sont incertaines, ne venant ni des mots ni des choses mais de leur rencontre inopinée dans un sujet déchiré entre sa singularité et son altérité, entre sa réflexion et sa communication. Juge ultime, il les entretient et s'y mire. C'est à lui qu'elles ressemblent le plus, à lui qui jamais ne s'avouera arbitraire.

\section{François Latraverse Université du Québec à Montréal}

44. "Words so connected as that they become signs of our thoughts, are called speech, of which every part is a name. But seeing (as is said) both marks and signs are necessary for the acquiring of philosophy, (marks by which we can remember our own thoughts, and signs by which we may make our thoughts known to others), names do both these offices; but they serve for marks before they are used as signs [...] So that the nature of a sign consist principally in this, that it is a mark taken for memory's sake; but it serves also by accident to signify and make known to others what we remember ourselves" (Th. Hobbes, Elements of philosophy, l.ii.3). 